\title{
ABSOLUTE TAUBERIAN CONSTANTS FOR CESÁRO MEANS OF A FUNCTION
}

BY

\section{SORAYA SHERIF}

ABSTRACT. This paper is concerned with introducing two estimates of the forms $F \leqslant C A_{k}(\alpha), F \leqslant D B_{k}(\alpha),(\alpha>0)$, where $F=\int_{0}^{\infty}\left|d\left\{f(\alpha x)-\sigma_{k}(x)\right\}\right|$, $\sigma_{k}(x)$ denote the Cesàro transform of order $k$ of the function $f(x)=\int_{0}^{x} g(t) d t$, $g(t)$ is a function of bounded variation in every finite interval of $t \geqslant 0, A_{k}(\alpha)$, $B_{k}(\alpha)$ are absolute Tauberian constants, $C=\int_{0}^{\infty}|d\{\operatorname{tg}(t)\}|<\infty, D=\int_{0}^{\infty}|d\{\phi(t)\}|$ $<\infty$ and $\phi(t)=t^{-1} \int_{0}^{t} u g(u) d u$. The constants $A_{k}(\alpha), B_{k}(\alpha)$ will be determined.

1. Introduction. We define the absolute summability by the Cesàro method of a function as follows:

DEFINITION 1. If

$$
\sigma_{k}(x)=\frac{k}{x^{k}} \int_{0}^{x}(x-t)^{k-1} f(t) d t, \quad(k \geqslant 0),
$$

then the function $f(x)$ is said to be absolutely summable $(C, k)$ or summable $|C, k|$ to $\sigma$ if $\sigma_{k}(x)$ is of bounded variation in $(0, \infty)$, and if it tends to $\sigma$ as $x \rightarrow \infty$.

This is the analogous definition of absolute summaility $(C, k)$ of the sequence $\left\{s_{n}\right\}$ given by Fekete [4] , and which is as follows.

DEFINITION 2. If $s_{n}=a_{0}+a_{1}+\cdots+a_{n}$, and

$$
C_{n}^{(k)}=\left(\begin{array}{c}
n+k \\
n
\end{array}\right)^{-1} \sum_{\nu=0}^{n}\left(\begin{array}{c}
n-\nu+k-1 \\
n-\nu
\end{array}\right) s_{\nu}, \quad(n=0,1, \ldots),
$$

then we say that the sequence $\left\{s_{n}\right\}$ is absolutely summable $(C, k)$ or summable $|C, k|$, if the sequence $\left\{C_{n}^{(k)}\right\}$ is of bounded variation, i.e. if

where

$$
\sum_{n=1}^{\infty}\left|\Delta C_{n}^{(k)}\right|<\infty
$$

$$
\Delta C_{n}^{(k)}=C_{n}^{(k)}-C_{n-1}^{(k)} .
$$

In Sherif [7], we introduced estimates of the forms

Received by the editors July $23,1974$.

AMS (MOS) subject classifications (1970). Primary 40D10, $40 \mathrm{G05}$. 
and

$$
\sum\left|\Delta\left(C_{n}^{(k)}-s_{n}\right)\right| \leqslant K \sum\left|\Delta\left(n a_{n}\right)\right|,(1)
$$

$$
\sum\left|\Delta\left(C_{n}^{(k)}-s_{n}\right)\right| \leqslant B \sum_{n}\left|\Delta\left(\frac{1}{n} \sum_{\nu=1}^{n-1} \nu a_{\nu}\right)\right|,
$$

where the sequence $\left\{n a_{n}\right\}$ is assumed to be either absolutely convergent or, more generally, absolutely summable $(C, 1)$; that is to say where we suppose that either

$$
\sum\left|\Delta\left(n a_{n}\right)\right|<\infty, \text { or } \sum_{n}\left|\left(\frac{1}{n} \sum_{\nu=1}^{n-1} v a_{\nu}\right)\right|<\infty,
$$

respectively; $k, B$ are absolute Tauberian constants.

Estimates of a similar type have been obtained by various authors which appear in the bibliography of Sherif [7], but for different summability methods instead, see for example Agnew [1], [2], Delange [3], Tenenbaum [9], and Sherif [5], [6].

In $\S \S 2,3$ of this paper, we consider the function-to-function absolute Cesàro summability. Our estimates will be of the forms

$$
\int_{0}^{\infty}\left|d\left\{f(\alpha x)-\sigma_{k}(x)\right\}\right| \leqslant A_{k}(\alpha) \int_{0}^{\infty}|d\{\operatorname{tg}(t)\}|, \quad(\alpha>0),
$$

and

$$
\int_{0}^{\infty}\left|d\left\{f(\alpha x)-\sigma_{k}(x)\right\}\right| \leqslant B_{k}(\alpha) \int_{0}^{\infty}|d \phi(t)|, \quad(\alpha>0),
$$

where $\sigma_{k}(x)$ is defined by $(1.1), g(t)$ is of bounded variation in every finite interval of $t \geqslant 0$,

$$
\begin{gathered}
f(x)=\int_{0}^{x} g(t) d t, \\
\phi(t)=\frac{1}{t} \int_{0}^{t} u g(u) d u,
\end{gathered}
$$

$A_{k}(\alpha), B_{k}(\alpha)$ are absolute Tauberian constants, and where we now assume that the function $\{\operatorname{tg}(t)\}$ either tends to a limit absolutely or, more generally, $a b$ solutely summable $(C, 1)$; that is to say where we suppose that either

$$
\int_{0}^{\infty}|d\{\operatorname{tg}(t)\}|<\infty,
$$

or

$$
\int_{0}^{\infty}|d\{\phi(t)\}|<\infty
$$

respectively.

(1) Unless otherwise indicated, the symbol $\Sigma$ stands for $\Sigma_{0}^{\infty}$. 
A few words of explanation may be given as to why we have taken $\alpha$ as arbitrary here while in [7] we considered only the analogue for series of the special case $\alpha=1$. In theorems like those of [1] and [2] where estimates for quantities such as $\left\{t_{m}-s_{n}\right\}\left(^{2}\right)$ are given ( $m$ being a given function of $n$ ), it is enough to suppose that $m / n \rightarrow \alpha$. In the theorems of [7] where we consider sums like $\Sigma\left|\Delta\left(t_{m}-s_{n}\right)\right|$, it is not enough to specify the limit of $m / n$ since, roughly speaking a small change in $m$ for all large $n$ may involve a large change in the sum of the differences. Moreover, we cannot suppose that $m=\alpha n$ since $n, m$ are required to be integers. But in considering expressions like $\int_{0}^{\infty}|d\{f(y)-\sigma(x)\}|$ where $\sigma(x)$ is a linear transform of a function $f(y), y, x$ can take all values, and we may therefore suppose that $y=\alpha x$.

I would like to express my gratitude to Professor B. Kuttner for his valuable suggestions.

2. Theorem 2.1. Suppose that (1.1), (1.4) and (1.6) hold. Then (1.2) holds, where for $k \geqslant 0$,

$$
A_{k}(\alpha)=\left\{\begin{array}{l}
\int_{1}^{1 / \alpha}\left\{\frac{(t-1)^{k}}{t^{k+1}}\right\} d t+\int_{1 / \alpha}^{\infty}\left\{\frac{t^{k}-(t-1)^{k}}{t^{k+1}}\right\} d t, \\
\log \alpha+\gamma+\frac{\Gamma^{\prime}(k+1)}{\Gamma(k+1)}, \text { for }(\alpha \leqslant 1),
\end{array}\right.
$$

( $\gamma$ is Euler's constant).

This result is the best possible in the sense that (1.2) becomes false if $A_{k}(\alpha)$ is replaced by any smaller constant.

For fixed $k$, the least possible value of $A_{k}(\alpha)$ occurs when

$$
\alpha=\alpha_{k}=1-1 / 2^{1 / k} \text {. }
$$

We remark that in (2.1), the two expressions given for $A_{k}(\alpha)$ both apply in the case $\alpha=1$; it is clear that they give the same value in this case.

For the proof of Theorem 2.1, we require the following lemma.

LEMMA 2.1. Suppose that

$$
G(x)=\int_{0}^{\infty} \chi(x, u) d h(u),
$$

where $\chi(x, u)$ is, for fixed $x, a$ continuous function of $u$ which vanishes for all sufficiently large $u$, and $h(u)$ is of bounded variation in every finite interval of $t \geqslant 0$. Then

$\left.{ }^{2}\right) t_{m}$ is a linear transform $T$. 


$$
\int_{0}^{\infty}|d G(x)| \leqslant A \int_{0}^{\infty}|d h(u)|,
$$

where

$$
A=\sup _{0<u} \int_{0}^{\infty}\left|d_{x} \chi(x, u)\right|,
$$

and this constant $A$ is the best possible in the sense that (2.3) becomes false if $A$ is replaced by any smaller constant.

The first part of Lemma 2.1 generalises a lemma given by Tatchell [8, Lemma 1], to which it reduces in the special case in which $h(u)$ is an indefinite integral, say $h(u)=\int g(u) d u$. On examining Tatchell's proof, it is seen that the more general result given here can be established by essentially the same argument. We now prove the second part of Lemma 2.1. Given $\epsilon>0$, we can choose $u_{0}$ such that

$$
\int_{0}^{\infty}\left|d_{x} \chi\left(x, u_{0}\right)\right|>A-\epsilon .
$$

Take

$$
h(u)= \begin{cases}0, & \text { for }\left(u<u_{0}\right) \\ 1, & \text { for }\left(u \geqslant u_{0}\right)\end{cases}
$$

Then

$$
G(x)=\chi\left(x, u_{0}\right)>0
$$

so that

$$
\int_{0}^{\infty}|d G(x)|>A-\epsilon,
$$

but

$$
\int_{0}^{\infty}|d h(u)|=1 .
$$

The conclusion thus follows.

Proof of Theorem 2.1. Using (1.4), we have

$$
f(\alpha x)=\int_{0}^{\alpha x} g(t) d t .
$$

But

$$
g(t)=\frac{1}{t} \int_{0}^{t} d(u g(u)) .
$$

Thus

$$
\begin{aligned}
f(\alpha x) & =\int_{0}^{\alpha x} \frac{d t}{t} \int_{0}^{t} d(u g(u))=\int_{0}^{\alpha x} d(u g(u)) \int_{u}^{x} \frac{d t}{t} \\
& =\int_{0}^{\alpha x}\left(\log \left(\frac{\alpha x}{u}\right)\right) d(u g(u)) .
\end{aligned}
$$


Also, on integrating (1.1) by parts, we have

$$
\sigma_{k}(x)=\frac{1}{x^{k}} \int_{0}^{x}(x-t)^{k} g(t) d t .
$$

Using (2.5) we get

$$
\begin{aligned}
\sigma_{k}(x) & =\frac{1}{x^{k}} \int_{0}^{x}(x-t)^{k} \frac{d t}{t} \int_{0}^{t} d(u g(u)) \\
& =\frac{1}{x^{k}} \int_{0}^{x} d(u g(u)) \int_{u}^{x}(x-t)^{k} \frac{d t}{t}
\end{aligned}
$$

It thus follows from (2.6) and (2.8) that

$$
f(\alpha x)-\sigma_{k}(x)=\int_{0}^{\alpha x}\left(\log \left(\frac{\alpha x}{u}\right)\right) d(u g(u))
$$

$$
-\frac{1}{x^{k}} \int_{0}^{x} d(u g(u)) \int_{u}^{x}(x-t)^{k} \frac{d t}{t}
$$

Now, (2.9) is a transformation of the type considered in Lemma 2.1. Thus, when $\alpha \leqslant 1$, the $\chi(x, u)$ of Lemma 2.1 is equal to

$$
\left\{\begin{array}{l}
\log \left(\frac{\alpha x}{u}\right)-\frac{1}{x^{k}} \int_{u}^{x}(x-t)^{k} \frac{d t}{t}, \text { for }(0<u<\alpha x), \\
-\frac{1}{x^{k}} \int_{u}^{x}(x-t)^{k} \frac{d t}{t}, \text { for }(\alpha x<u<x) \\
0, \text { for }(u>x)
\end{array}\right.
$$

When $\alpha \geqslant 1$, the $\chi(x, u)$ of Lemma 2.1 is equal to

$$
\left\{\begin{array}{l}
\log \left(\frac{\alpha x}{u}\right)-\frac{1}{x^{k}} \int_{u}^{x}(x-t)^{k} \frac{d t}{t}, \text { for }(0<u<x) \\
\log (\alpha x / u), \text { for }(x<u<\alpha x) \\
0, \text { for }(u>\alpha x)
\end{array}\right.
$$

Hence, when $\alpha \leqslant 1, \partial \chi(x, u) / \partial x$ is equal to

$$
\left\{\begin{array}{l}
1 / x-(x-u)^{k} / x^{k+1}, \quad \text { for }(0<u<\alpha x) \\
-(x-u)^{k} / x^{k+1}, \text { for }(\alpha x<u<x) \\
0, \text { for }(u>x) .
\end{array}\right.
$$


When $\alpha \geqslant 1, \partial \chi(x, u) / \partial x$ is equal to

$$
\left\{\begin{array}{l}
1 / x-(x-u)^{k} / x^{k+1}, \text { for }(0<u<x), \\
1 / x, \text { for }(x<u<\alpha x), \\
0, \text { for }(u>\alpha x) .
\end{array}\right.
$$

Applying Lemma 2.1, it follows from (2.10) and (2.11) that

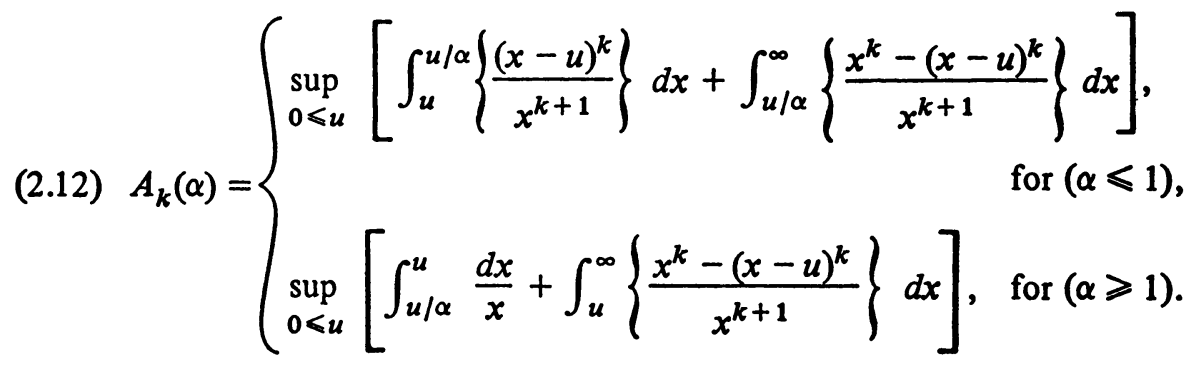

Putting $x=u t$, it is easily seen that (2.12) becomes

$$
A_{k}(\alpha)=\left\{\begin{array}{l}
\int_{1}^{1 / \alpha}\left\{\frac{(t-1)^{k}}{t^{k+1}}\right\} d t+\int_{1 / \alpha}^{\infty}\left\{\frac{t^{k}-(t-1)^{k}}{t^{k+1}}\right\} d t, \\
\text { for }(\alpha \leqslant 1), \\
\log \alpha+\int_{0}^{\infty}\left\{\frac{t^{k}-(t-1)^{k}}{t^{k+1}}\right\} d t, \text { for }(\alpha \geqslant 1) .
\end{array}\right.
$$

Now

$$
\int_{1}^{\infty}\left\{\frac{t^{k}-(t-1)^{k}}{t^{k+1}}\right\} d t=\lim _{\delta \rightarrow 0+}\left[\int_{1}^{\infty}\left\{\frac{t^{k-\delta}-(t-1)^{k-\delta}}{t^{k+1}}\right\} d t\right]
$$

$$
\begin{aligned}
& =\lim _{\delta \rightarrow 0+}\left[\int_{1}^{\infty} t^{-\delta-1} d t-\int_{1}^{\infty}\left\{\frac{(t-1)^{k-\delta}}{t^{k+1}}\right\} d t\right] \\
& =\lim _{\delta \rightarrow 0+}\left[\frac{1}{\delta}-\int_{1}^{\infty}(1-u)^{k-\delta} u^{\delta-1} d u\right] .
\end{aligned}
$$

But

(2.15) $\int_{0}^{1}(1-u)^{k-\delta} u^{\delta-1} d u=B(k-\delta+1, \delta)=\Gamma(k-\delta+1) \Gamma(\delta) / \Gamma(k+1)$,

(2.16) $\Gamma(\delta)=\Gamma(\delta+1) / \delta=\delta^{-1}\left[\Gamma(1)+\delta \Gamma^{\prime}(1)+O\left(|\delta|^{2}\right)\right]=\delta^{-1}-\gamma+O(\delta)$,

and 


$$
\Gamma(k-\delta+1)=\Gamma(k+1)-\delta \Gamma^{\prime}(k+1)+O\left(\delta^{2}\right) .
$$

Combining (2.14)-(2.17), it follows that

$$
\int_{1}^{\infty}\left\{\left(t^{k}-(t-1)^{k}\right) / t^{k+1}\right\} d t=\gamma+\Gamma^{\prime}(k+1) / \Gamma(k+1) .
$$

Substituting (2.18) in (2.13), the result that (1.2) holds, with $A_{k}(\alpha)$ given by (2.1), together with the assertion that this is the best possible result follow at once from Lemma 2.1 .

We now prove equation (2.2). It is clear that $A_{k}(\alpha)$ defined by (2.1) is increasing for $\alpha \geqslant 1$. We may therefore restrict our consideration to the case $\alpha \leqslant 1$. Thus,

$$
\begin{aligned}
\frac{\partial}{\partial \alpha} A_{k}(\alpha) & =-\frac{1}{\alpha^{2}}\left\{\alpha^{k+1}\left(\frac{1}{\alpha}-1\right)^{k}-\alpha^{k+1}\left(\left(\frac{1}{\alpha}\right)^{k}-\left(\frac{1}{\alpha}-1\right)^{k}\right)\right\} \\
& =\alpha^{-1}\left\{1-2(1-\alpha)^{k}\right\} .
\end{aligned}
$$

It is then clear that the expression in curly brackets of (2.19) is negative when $(1-\alpha)^{k}>1 / 2$ and is positive when $(1-\alpha)^{k}<1 / 2$. It vanishes when $(1-\alpha)^{k}=1 / 2$ from which (2.2) holds.

3. THEOREM 3.1. Suppose that (1.1), (1.4), (1.5), and (1.7) hold. Then (1.3) holds, where for

(i) $k \geqslant 1$,

$$
B_{k}(\alpha)=\left\{\begin{array}{l}
A_{k}(\alpha)+2(1-\alpha)^{k}, \quad \text { for }(\alpha \leqslant 1), \\
A_{k}(\alpha), \quad \text { for }(\alpha \geqslant 1)
\end{array}\right.
$$

(ii) $k \leqslant 1$,

$$
B_{k}(\alpha)=\left\{\begin{array}{r}
2+\int_{1}^{1 / \alpha}\left\{\frac{(t-1)^{k}}{t^{k+1}}\right\} d t+\int_{1 / \alpha}^{\infty}\left\{\frac{(t-1)^{k}-t^{k}}{t^{k+1}}\right\} d t, \\
\text { for }(\alpha \leqslant 1), \\
-\left[\gamma+\Gamma^{\prime}(k+1) /(k+1)\right]+2+\log \alpha, \text { for }(\alpha \geqslant 1) .
\end{array}\right.
$$

This result is the best possible in the sense that (1.3) becomes false if $B_{k}(\alpha)$ is replaced by any smaller constant.

For fixed $k$, the least possible value of $B_{k}(\alpha)$ occurs when $\alpha=\alpha_{k}$ where $\alpha_{k}$ is defined as follows:

(a) If $k \geqslant 1$, then $\alpha_{k}$ is the unique solution in the range $0<\alpha<1$ of

$$
1-2(1-\alpha)^{k}-2 k \alpha(1-\alpha)^{k-1}=0 .
$$

(b) If $k \leqslant 1$, then $\alpha_{k}=1$. 
We remark that in (3.1) the two expressions given for $B_{k}(\alpha)$ both apply in the case $\alpha=1$; it is clear that they give the same value in this case. An analogous remark applies to (3.2). In a similar way, (i) and (ii) both apply when $k=1$. Further, so do (a) and (b).

Proof. Using (1.5), we see that $\phi(u)$ is an indefinite integral of the function $\phi^{\prime}(u)$ defined by

$$
\phi^{\prime}(u)=g(u)-\frac{1}{u^{2}} \int_{0}^{u} \operatorname{tg}(t) d t .
$$

Thus,

$$
g(u)=\phi^{\prime}(u)+\phi(u) / u=\phi^{\prime}(u)+\frac{1}{u} \int_{0}^{u} \phi^{\prime}(y) d y .
$$

Using (3.4), it follows from (2.4) that

$$
f(\alpha x)=\int_{0}^{\alpha x} \phi^{\prime}(u) d u+\int_{0}^{\alpha x} \frac{d w}{w} \int_{0}^{w} \phi^{\prime}(u) d u .
$$

Inverting the order of integration in the second integral of (3.5), and then evaluating the inner integral, we get

$$
f(\alpha x)=\int_{0}^{\alpha x}\left(1+\log \left(\frac{\alpha x}{u}\right)\right) \phi^{\prime}(u) d u .
$$

Also, using (3.4) it follows from (2.7) that

$$
\begin{aligned}
\sigma_{k}(x)= & \frac{1}{x^{k}} \int_{0}^{x}(x-u)^{k} \phi^{\prime}(u) d u \\
& +\frac{1}{x^{k}} \int_{0}^{x}(x-y)^{k} \quad \frac{d y}{y} \int_{0}^{y} \phi^{\prime}(u) d u .
\end{aligned}
$$

Inverting the order of integration in the second integral of (3.7), we get

$$
\begin{aligned}
\sigma_{k}(x)= & \frac{1}{x^{k}} \int_{0}^{x}(x-u)^{k} \phi^{\prime}(u) d u \\
& +\frac{1}{x^{k}} \int_{0}^{x} \phi^{\prime}(u) d u \int_{u}^{x}(x-y)^{k} \frac{d y}{y}
\end{aligned}
$$

Thus, it follows from (3.6) and (3.8) that

$$
\begin{aligned}
f(\alpha x)-\sigma_{k}(x)= & \int_{0}^{\alpha x}\left(1+\log \left(\frac{\alpha x}{u}\right)\right) \phi^{\prime}(u) d u \\
& -\frac{1}{x^{k}} \int_{0}^{x}\left[(x-u)^{k}+\int_{u}^{x}(x-y)^{k} \frac{d y}{y}\right] \phi^{\prime}(u) d u .
\end{aligned}
$$

Now (3.9) is a transformation of the type considered in Lemma 2.1. Thus, when $\alpha \leqslant 1$, the $\chi(x, u)$ of Lemma 2.1 is equal to 


$$
\left\{\begin{array}{l}
1+\log \left(\frac{\alpha x}{u}\right)-\frac{1}{x^{k}}\left[(x-u)^{k}+\int_{u}^{x}(x-y)^{k} \frac{d y}{y}\right], \text { for }(0<u<\alpha x), \\
-\frac{1}{x^{k}}\left[(x-u)^{k}+\int_{u}^{x}(x-y)^{k} \frac{d y}{y}\right], \text { for }(\alpha x<u<x), \\
0, \text { for }(u>x) .
\end{array}\right.
$$

When $\alpha \geqslant 1$, the $\chi(x, u)$ of Lemma 2.1 is equal to

$$
\left\{\begin{array}{l}
1+\log \left(\frac{\alpha x}{u}\right)-\frac{1}{x^{k}}\left[(x-u)^{k}+\int_{u}^{x}(x-y)^{k} \frac{d y}{y}\right], \text { for }(0<u<x), \\
1+\log (\alpha x / u), \text { for }(x<u<\alpha x), \\
0, \text { for }(u>\alpha x) .
\end{array}\right.
$$

Hence, when $\alpha \leqslant 1, \partial \chi(x, u) / \partial x$ is equal to

$$
\left\{\begin{array}{l}
D_{k}(x, u) / x^{k+1}, \text { (say) for }(0<u<\alpha x) \\
-(x-u)^{k} / x^{k+1}-k u(x-u)^{k-1} / x^{k+1}, \text { for }(\alpha x<u<x) \\
0, \text { for }(u>x)
\end{array}\right.
$$

where

$$
D_{k}(x, u)=x^{k}-k u(x-u)^{k-1}-(x-u)^{k} .
$$

When $\alpha \geqslant 1, \partial \chi(x, u) / \partial x$ is equal to

$$
\begin{cases}D_{k}(x, u) / x^{k+1}, & \text { for }(0<u<x), \\ 1 / x, & \text { for }(x<u<\alpha x), \\ 0, & \text { for }(u>\alpha x) .\end{cases}
$$

We further note that $\chi(x, u)$ has a jump of 1 at $u=\alpha x$ (but is continuous at $u=x$, except when $\alpha=1$ ). Applying Lemma 2.1, it follows that

$(3.11) B_{k}(\alpha)=\left\{\begin{aligned} & \sup _{0<u}\left[\int_{u}^{u / \alpha}\left\{(x-u)^{k}+k u(x-u)^{k-1} / x^{k+1}\right\} d x\right. \\ &\left.+\int_{u / \alpha}^{\infty}\left|D_{k}(x, u) / x^{k+1}\right| d x+1\right], \\ & \sup _{0<u}\left[\int_{u / \alpha}^{u} \frac{d x}{x}+\int_{u}^{\infty}\left|D_{k}(x, u) / x^{k+1}\right| d x+1\right], \text { for }(\alpha \leqslant 1), \\ & \text { for }(\alpha \geqslant 1) .\end{aligned}\right.$ 
Now, $D_{k}(x, u)$ vanishes when $u=0$. Also,

$$
\partial D_{k}(x, u) / \partial u=k(k-1) u(x-u)^{k-2} .
$$

So, if $k \geqslant 1, D_{k}(x, u) \geqslant 0$, and if $k \leqslant 1, D_{k}(x, u) \leqslant 0$.

(i) $k \geqslant 1$.

Since $D_{k}(x, u) \geqslant 0$, we can omit the modulus sign in (3.11). On putting $x=t u$, we find, for $\alpha \leqslant 1$,

$$
\begin{aligned}
B_{k}(\alpha) & =\int_{1}^{1 / \alpha}\left\{\frac{(t-1)^{k}+k(t-1)^{k-1}}{t^{k+1}}\right\} d t+\int_{1 / \alpha}^{\infty}\left\{\frac{D_{k}(t, 1)}{t^{k+1}}\right\} d t+1 \\
& =A_{k}(\alpha)+k\left\{\int_{1}^{1 / \alpha}-\int_{1 / \alpha}^{\infty}\right\}\left(\frac{(t-1)^{k-1}}{t^{k+1}}\right) d t+1
\end{aligned}
$$

For $\alpha \geqslant 1$, we have

$$
\begin{aligned}
B_{k}(\alpha) & =\log \alpha+\int_{1}^{\infty}\left\{D_{k}(t, 1) / t^{k+1}\right\} d t+1 \\
& =A_{k}(\alpha)-k \int_{1}^{\infty}\left\{(t-1)^{k-1} / t^{k+1}\right\} d t+1
\end{aligned}
$$

Now,

$$
k \int_{1}^{\infty}\left\{(t-1)^{k-1} / t^{k+1}\right\} d t=k \int_{0}^{1}(1-u)^{k-1} d u=1
$$

Substituting (3.14) in (3.12), we get

$$
B_{k}(\alpha)=A_{k}(\alpha)+2 k \int_{1}^{1 / \alpha} d\left(\frac{u-1}{u}\right)^{k}, \text { for }(\alpha \leqslant 1),
$$

from which the first expression of (3.1) follows. The second expression of (3.1) follows at once from (3.13) and (3.14).

(ii) $k \leqslant 1$.

Since $D_{k}(x, u) \leqslant 0$, it is clear that (3.11) becomes

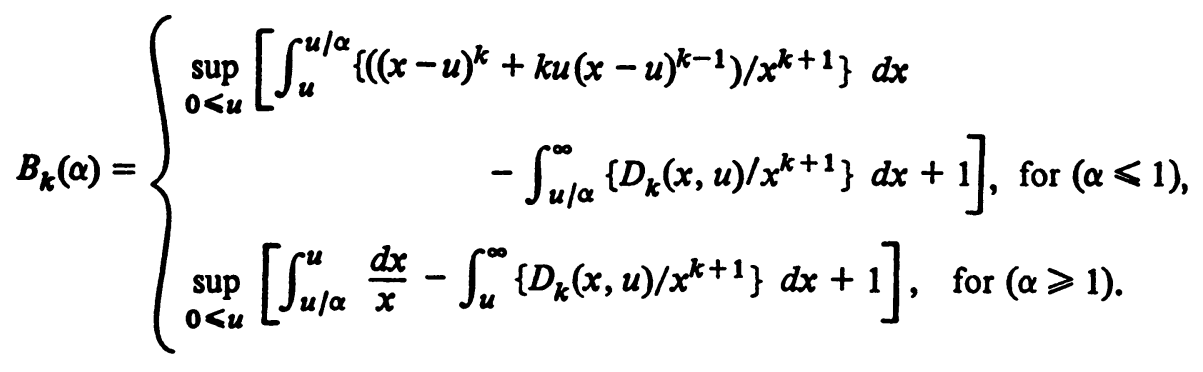

On putting $x=u t$, we find that, for $\alpha \leqslant 1$, 


$$
\begin{aligned}
B_{k}(\alpha)= & \int_{1}^{1 / \alpha}\left\{\frac{(t-1)^{k}+k(t-1)^{k-1}}{t^{k+1}}\right\} d t-\int_{1 / \alpha}^{\infty}\left\{\frac{D_{k}(t, 1)}{t^{k+1}}\right\} d t+1 \\
= & \int_{1}^{1 / \alpha}\left\{\frac{(t-1)^{k}}{t^{k+1}}\right\} d t+k \int_{1}^{\infty}\left\{\frac{(t-1)^{k-1}}{t^{k+1}}\right\} d t \\
& +\int_{1 / \alpha}^{\infty}\left\{\frac{(t-1)^{k}-t^{k}}{t^{k+1}}\right\} d t+1
\end{aligned}
$$

Thus, the first expression of (3.2) follows at once from (3.14) and (3.16). For $\alpha \geqslant 1$, we have

$$
B_{k}(\alpha)=\log \alpha-\int_{1}^{\infty}\left\{D_{k}(t, 1) / t^{k+1}\right\} d t+1
$$

Substituting (3.14) in (3.17) it follows that

$$
B_{k}(\alpha)=\log \alpha+2-\int_{1}^{\infty}\left\{\left(t^{k}-(t-1)^{k}\right) / t^{k+1}\right\} d t
$$

Hence, the second expression of (3.2) follows from (2.18) and (3.18). It is thus clear that the result $(1.3)$ with $B_{k}(\alpha)$ given by $(3.1)$ for $(k \geqslant 1)$ and by (3.2) for $(k \leqslant 1)$ together with the assertion that this is the best possible result follow at once from Lemma 2.1 .

We now prove (a). When $\alpha \geqslant 1$, it is clear that $B_{k}(\alpha)$ defined by (3.1) is increasing. We may therefore restrict our consideration to the case $\alpha \leqslant 1$. Thus

$$
\begin{aligned}
\frac{\partial}{\partial \alpha} B_{k}(\alpha) & =\frac{1}{2}-\frac{2}{\alpha}(1-\alpha)^{k}-\frac{2 k}{\alpha^{2}}-\left\{\alpha^{k+1}\left(\frac{1}{\alpha}-1\right)^{k-1}\right\} \\
& =(1 / \alpha)\left\{1-2(1-\alpha)^{k}-2 k \alpha(1-\alpha)^{k-1}\right\}
\end{aligned}
$$

When $k \geqslant 1$, then we can verify by differentiation that the expression in curly brackets in (3.19) is increasing for $0<\alpha \leqslant 1$. It tends to 1 as $\alpha \rightarrow 1$ and to -1 as $\alpha \rightarrow 0$, and has thus a unique zero (say $\alpha=\alpha_{k}$ ) for $0<\alpha \leqslant 1$, and the minimum of $B_{k}(\alpha)$ occurs when $\alpha=\alpha_{k}$ is the unique solution in the range 0 $<\alpha \leqslant 1$ of equation (3.3). To prove (b) it is clear that in the case $k \leqslant 1$, $B_{k}(\alpha)$ defined by (3.2) is decreasing for $0<\alpha<1$; and when $k<1, \alpha \geqslant 1$, $B_{k}(\alpha)$ is increasing. Thus, the least possible value of $B_{k}(\alpha)$ occurs when $\alpha=1$.

\section{REFERENCES}

1. R. P. Agnew, Integral transformations and Tauberian constants, Trans. Amer. Math. Soc. 72 (1952), 501-518. MR 13, 934.

2. - Tauberian relations among partial sums, Riesz transforms, and Abel transforms of series, J. Reine Angew. Math. 193 (1954), 94-118. MR 16, 237. 
3. H. Delange, Sur les théorèmes inverses des procédès de sommation des séries divergentes. I, Ann. Sci. École Norm. Sup. (3) 67 (1950), 99-160. MR 12, 253.

4. M. Fekete, Vizsgálatok az absolut summabilis sorokral, alkalmázassal a Dirichletés Fourier-sorokra, Math. és Termesz. Ert. 32 (1914), 389-425.

5. S. Sherif, Tauberian constants for general triangular matrices and certain special types of Hausdorff means, Math. Z. 89 (1965), 312-323. MR 31 \# 5013.

6. - A Tauberian constant for the $\left(S, \mu_{n+1}\right)$ transformation, Tôhoku Math. J. (2) 19 (1967), 110-125. MR 36 \# 1877.

7. - Absolute Tauberian constants for Cesàro means, Trans. Amer. Math. Soc. 168 (1972), 233-241. MR 45 \# 4013.

8. J. B. Tatchell, $A$ theorem on absolute Riesz summability, J. London Math. Soc. 29 (1954), 49-59. MR 15, 305.

9. M. T. Tenenbaum, Transforms of Tauberian series by Riesz methods of different orders, Duke Math. J. 25 (1958), 181-191. MR 20 \# 193.

DEPARTMENT OF MATHEMATICS, EDUCATION COLLEGE FOR WOMEN, RIYADH, SAUDI ARABIA 